\title{
Sobre los usos y la cronología de las pizarras numerales: Reflexiones a partir del caso del yacimiento de Valdelobos (Montijo, Badajoz)
}

\section{Use and chronology of numeral slates: Some remarks on the case of the archaeological site of Valdelobos (Montijo, Badajoz)}

\author{
Tomás Cordero Ruiz \\ Instituto de Arqueología de Mérida (CSIC-Gobierno de Extremadura) \\ Iñaki Martín Viso \\ Departamento de Historia Medieval, Moderna y Contemporánea. Universidad de Salamanca
}

\section{RESUMEN}

En este trabajo pretendemos ofrecer algunas reflexiones sobre el uso, función y cronología de las llamadas pizarras numerales visigodas con el fin de ofrecer nuevas perspectivas de análisis que ayuden a su mejor comprensión. Para ello tomamos como referencia principal la pizarra hallada recientemente en el yacimiento de Valdelobos, inserto dentro del ager Emeritensis, pero sin descuidar su relación con los ejemplares documentados en el sector suroccidental de la cuenca del Duero y en la zona meridional del Sistema Central; región de donde procede la inmensa mayoría de las pizarras conocidas.

\section{SUMMARY}

In this paper we reflect on the use, function and chronology of the so-called 'Visigothic numeral' slates in order to offer new perspectives of analysis and to achieve a better understanding of them. Our main reference is the recently found slate from Valdelobos archaeological site, which is comprised within the ager Emeritensis, but also takes into account its relation to other similar items documented in the areas located in the southwestern-section of the Duero basin and the southern-part of the mountains of the Central System where most of the slates have been found.

PALABRAS CLAVE: Antigüedad Tardía, pizarras numerales, mundo rural, ager Emeritensis.

KEYWORDS: Late Antiquity, numeral slates, rural world, ager Emeritensis.

1. EL YACIMIENTO DE VALDELOBOS Y SU INSERCIÓN EN EL MARCO DEL TERRITORIO EMERITENSE

Las pizarras con signos numerales son un tipo de hallazgo frecuente en algunas áreas del suroeste de la cuenca del Duero y del piedemonte meridional del Sistema Central. Conocidas desde finales del siglo XIX, su comprensión continúa siendo oscura, ya que ofrecen una información que se reduce a una serie de cifras, sin mayores indicaciones. A ello se añade la ausencia de contextos arqueológicos claros que permitan entender adecuadamente sus usos y cronología. Sin embargo, el alto número de ejemplares recuperados - a falta de un elenco detallado puede situarse por encima de los $2000^{1}$ - obliga a plantearse algunas consideraciones a fin de encuadrar este material dentro de un marco explicativo que supere la mera descripción del hallazgo como tal. Nuestra intención no es resolver todas las dudas acerca de las pizarras numerales sino ofrecer algunas reflexiones partiendo de un caso concreto, el del yacimiento de Valdelobos (Montijo, Badajoz), que ha sido recientemente objeto de una intervención arqueológica, por lo que contamos con una información actualizada; aquí se encontró una pizarra numeral que nos servirá para realizar ciertas apreciaciones. Resulta evidente que esta elección representa en sí misma una limitación, ya que es fácil pensar que un material tan abundante debió ser usado para funciones muy diversas y en situaciones heterogéneas. De todos modos, nos parece interesante efectuar un estudio detallado

\footnotetext{
${ }^{1}$ Carecemos todavía de una base de datos sistemática con todos los lugares con hallazgos de pizarras. Velázquez Soriano 2005a nos ofrece un elenco bastante amplio, pero no es ni pretende ser- un listado completo. En cualquier caso, sirve como referencia para comprobar la extensión de la geografía de las pizarras.
} 


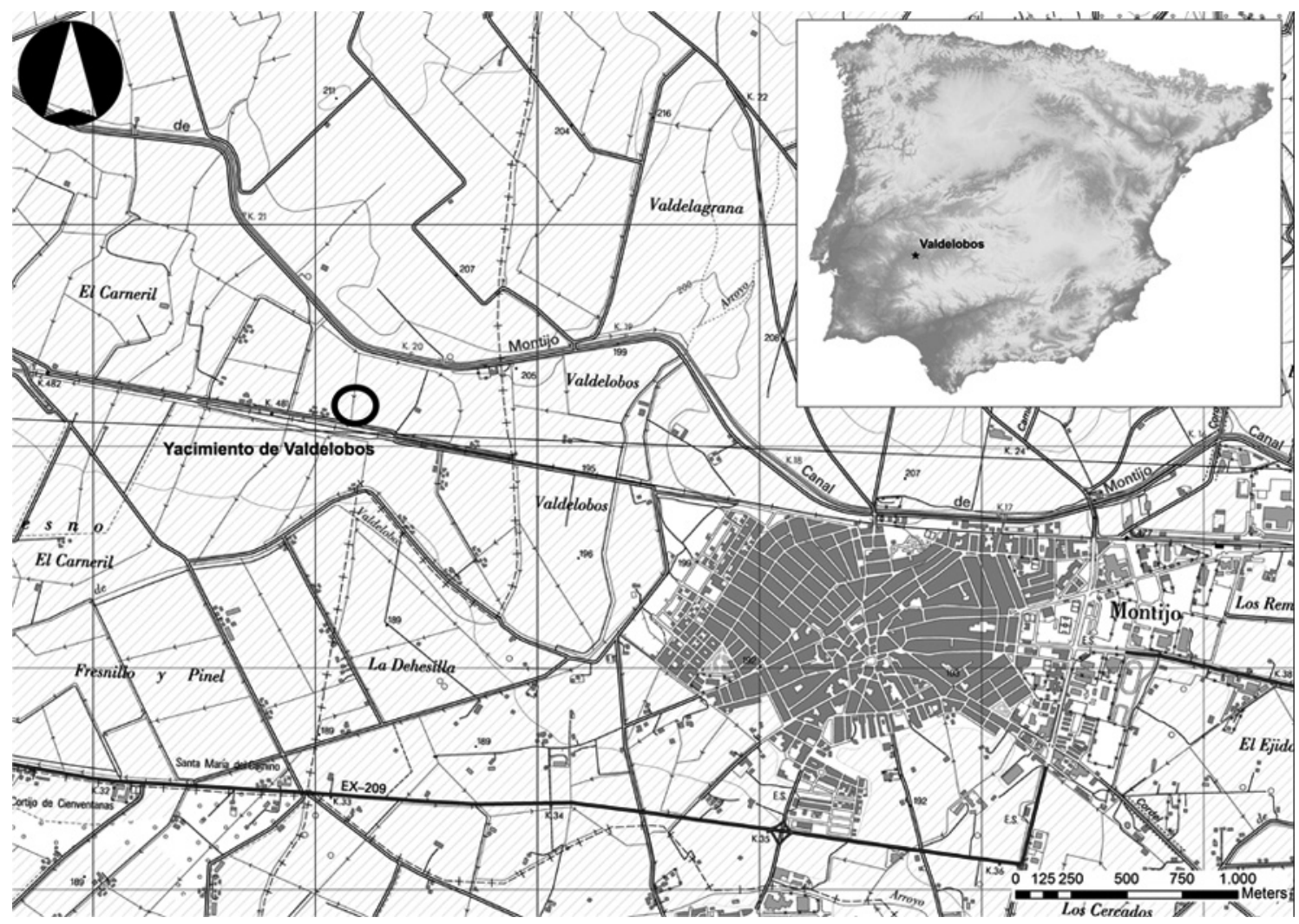

Figura 1. Localización del yacimiento de Valdelobos.

que permita quizá dar alguna respuesta y, sobre todo, abrir nuevas vías de investigación y de explicación.

La primera referencia sobre el yacimiento en cuestión procede del estudio de Gorges y Rodríguez Martín (2000: 129), quienes lo citan con el nombre de 'El Fresnillo'. Sin embargo, el topónimo de Valdelobos parece más correcto debido a la lejanía del primero con el lugar donde se concentran los restos arqueológicos documentados (Fig. 1). Ambos investigadores identificaron en superficie restos de fustes y bloques de granitos, placas de mármol, fragmentos de opus signinum, cerámica común, paredes finas procedentes de los talleres emeritenses, T. S. sudgálica, T. S. hispánica y T. S. africana. Además, advirtieron de la destrucción de un pavimento musivo de cronología tardía por las periódicas tareas agrícolas desarrolladas en el yacimiento, que dataron entre los siglos I y IV.

Posteriormente, las tareas de construcción de la nueva línea de tren AVE entre Madrid y Lisboa a su paso por el término municipal de Montijo propiciaron la realización de una intervención arqueológica en el sitio de Valdelobos, adjudicada a la empresa
Arqveochek S. L. U. y condicionada espacialmente por la traza de la nueva línea de ferrocarril. Los trabajos abarcaron una superficie total de $11000 \mathrm{~m}^{2}$, en los que se ha podido documentar una rica secuencia estratigráfica que comprende desde el siglo I al IX. ${ }^{2}$ No obstante, cabe destacar que las anteriores tareas de explanación y aterrazamiento llevadas a cabo en esta zona, destinadas a facilitar su aprovechamiento

${ }^{2}$ El yacimiento de Valdelobos ha sido excavado por dos grupos de arqueólogas/os diferentes. El primero, conformado por D. ${ }^{a}$ Yolanda Pereira y a D. ${ }^{a}$ Raquel Expósito, halló la pizarra numeral analizada en este trabajo. El segundo, compuesto por D. Fernando Sánchez, D. Diego Sanabria, D. Francisco Portalo, D. ${ }^{a}$ Renata Rosa y D. Víctor Gibello de la empresa Arqveochek S. L. U., continuó con la excavación posteriormente. Agradecemos a todos, especialmente a D. Fernando Sánchez, la información proporcionada. De otro lado, nos gustaría destacar las facilidades proporcionadas por el Museo Arqueológico Provincial de Badajoz para el estudio de la pieza, sobre todo a D. Manuel de Alvarado Gonzalo, su director en ese momento. Por último, significar la incidencia que ha tenido su excavación en la prensa regional, siendo referenciado en el periódico Hoy los días 10 de octubre de 2008 y 7 de septiembre de 2009 (ambas noticias pueden consultarse en su edición digital www.hoy.es). 


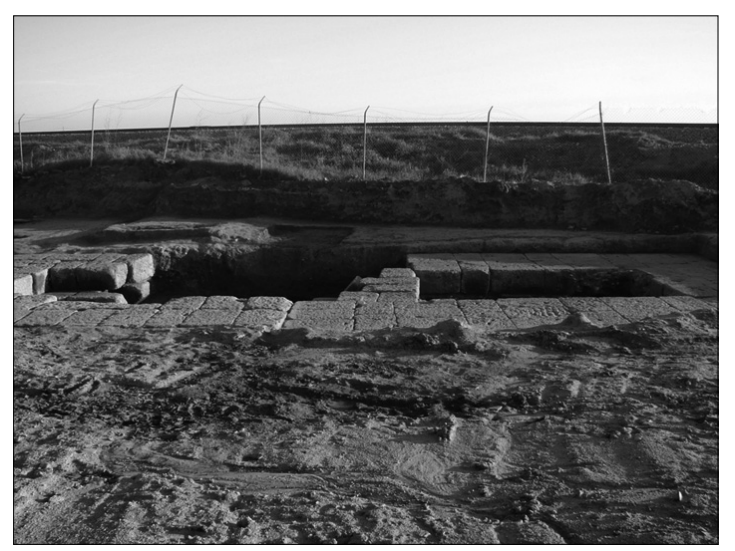

Figura 2. Mausoleo realizado en opus quadratum.

agrícola, han afectado en gran medida al grado de conservación de las estructuras documentadas.

Los restos más antiguos documentados, adscritos al período altoimperial, se corresponden con un mausoleo edificado en opus quadratum y un horno cerámico. El primero presenta dos cámaras funerarias, una de ellas, que presenta peor factura y reutiliza en su fábrica sillares de granito, se adosó en un momento posterior. Este edificio fue fuertemente expoliado en época emiral (Fig. 2).

El segundo, conserva su cámara de combustión realizada con ladrillos de adobe. No se han documentado estructuras de habitación de esta cronología, aunque si tenemos en cuenta la cantidad y la calidad de los materiales observados al Norte del límite septentrional del área excavada (Gorges y Rodríguez Martín 2000: 129), parece probable asumir que en ella se localizase la pars urbana de una villa. Establecer su cronología de un modo preciso es complicado debido a la falta de una intervención arqueológica en esta zona. No obstante, si tomamos como referentes temporales la presencia de cerámica emeritense de paredes finas y de un mosaico de cronología tardía junto a producciones de T. S. Africana C, podríamos asignarle como hipótesis de trabajo una cronología entre los siglos I y v.

En la parte occidental del área excavada se identificaron los restos de un edificio compuesto por una nave corrida y tres pasillos datados en el siglo IV, muy arrasados y conservados a nivel de cimentación, interpretados como los restos de un almacén relacionado con la posible pars urbana de la villa situada al Norte. Esta edificación presenta diferentes fases de ocupación, manteniendo siempre la función ya reseñada. La primera, la más complicada de documentar debido a la escasa cultura material conservada, se encuadraría en el período altoimperial. La segunda, asociada a la presencia de materiales como T.S hispánica tardía o lucernas Dressel 30, es datada en el siglo IV. Por último, cabría destacar una probable continuidad hasta un momento indeterminado del siglo $\mathrm{V}$, aunque esta fase no puede afirmarse con seguridad debido alto arrasamiento que presentaban los restos exhumados. Esta construcción fue amortizada por una necrópolis encuadrada entre los siglos vi y VII, sin que pueda descartarse un hiato temporal entre la última fase de uso del almacén y la disposición de las inhumaciones, que se extiende en hiladas bien definidas desde esta zona, hasta el mausoleo altoimperial mencionado anteriormente (Fig. 3).

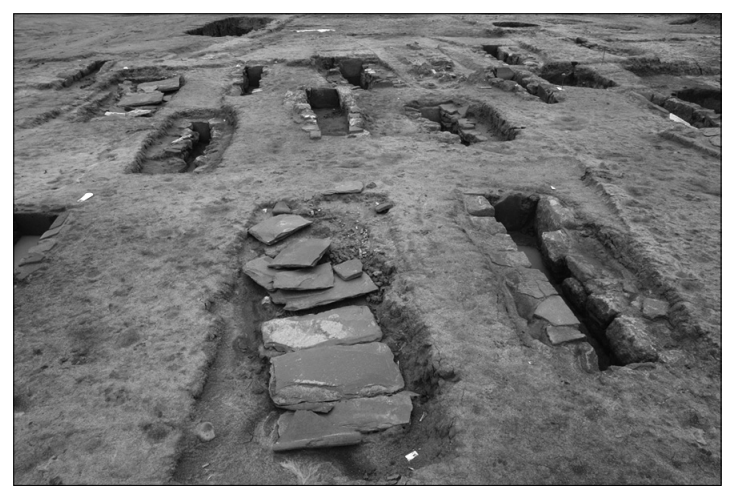

Figura 3. Detalle de la organización espacial de la necrópolis.

Las más antiguas se localizan en el espacio anexo al mausoleo. Esta disposición parece indicar que este monumento gozó de cierta preeminencia, probablemente fue el núcleo original de esta necrópolis.

La última fase de ocupación del yacimiento, datada entre los siglos VIII y IX, se identifica con el abandono de la necrópolis y su amortización por numerosos silos excavados en el suelo, con las paredes sin revocar, que no han podido ser relacionados con ninguna estructura de hábitat. Además, de diferentes zanjas de función indefinida, posibles basureros o fosas de expolio. En una de estas últimas, donde se documentó material cerámico que abarca desde el período romano al emiral, fue documentada la pizarra numeral objeto de este análisis ${ }^{3}$ (Fig. 4).

Con los datos expuestos, cabe remontar el origen de la ocupación de este lugar, localizado dentro del ager de Augusta Emerita (Cordero Ruiz 2010), al siglo I, posiblemente en su segunda mitad. Esta da-

\footnotetext{
${ }^{3}$ La pieza fue presentada como pieza del mes del Museo Arqueológico Provincial de Badajoz en mayo de 2009.
} 


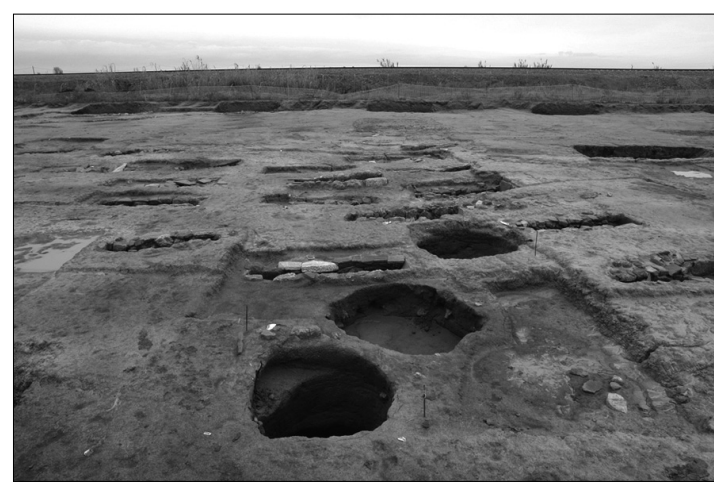

Figura 4. Vista general de los silos datados en época emiral.

tación se deduce de la documentación de cerámicas de paredes finas adscritas a los talleres emeritenses (Rodríguez Martín 1996: 179). Por otro lado, la presencia del mausoleo es otra de las importantes peculiaridades que presenta Valdelobos, ya que es uno de los pocos edificios altoimperiales de este tipo que se documentan fuera del ámbito periurbano emeritense (Márquez Pérez 2007).

Atendiendo al registro material de los yacimientos conocidos, parece que en el siglo i se produce una efectiva ocupación del territorio emeritense, en coincidencia con la implantación del sistema de villae. Concretamente en la cuenca media del Guadiana, donde se localiza el yacimiento de Valdelobos, encontramos un buen número de ejemplos cuyos orígenes pueden encuadrarse en esta centuria. En esta comarca, donde los suelos presentan óptimas condiciones para el desarrollo de la agricultura y la ganadería, el patrón de poblamiento parece estar articulado por las calzadas Alio Itinere ab Olisippone Emeritam e Iter ab Olisippone Emeritam, casi paralelas al curso del río Guadiana en este sector (Cordero Ruiz 2011: 550).

La necrópolis de Valdelobos guarda similitud con otros ejemplos conocidos en la cuenca media del Guadiana, concretamente en el tramo comprendido entre las actuales poblaciones de Mérida y Badajoz: Granja Céspedes (Matesanz Vera y Sánchez Fernández 2007), Torrebaja (Ramírez Sádaba 1991), Torre Águila (Rodríguez Martín 1997) o La Picuriña (Rubio Muñoz 1982). Sin embargo y al igual que en el caso que nos ocupa, no disponemos de suficiente documentación que permita asegurar una relación espacio-temporal entre estos espacios funerarios y un asentamiento concreto, solo podemos intuirla. No obstante, cabe destacar las grandes concomitancias existentes entre Valdelobos y los casos de Torre Águila o La Picuriña. En Torre Águila, la necrópo- lis dispuesta sobre la antigua villa (Rodríguez Martín 1997) donde no existen pruebas que nos permitan situar un edificio de culto cristiano (Mateos Cruz 2003: 117), podría indicarnos, si atendemos a su continuidad temporal (siglos VI y VII) y al gran número de inhumaciones documentadas, la presencia de un importante núcleo de población en las cercanías, quizás un vicus, o de diferentes núcleos más pequeños en los alrededores, una suerte de villulae. ${ }^{4}$ En la Picuriña, el estadio final de la necrópolis se encuadra en el siglo vil por la cronología de los ajuares funerarios documentados, aunque posteriormente, parece relacionarse con la presencia de silos circulares.

Esta realidad ¿podría indicarnos que nos encontramos ante necrópolis que actúan cómo centros funerarios de una población campesina residente en sus alrededores? La información disponible no permite ni confirmar ni descartar esta posibilidad. En primer lugar, el conocimiento de los yacimientos mencionados procede de excavaciones antiguas o de modernas intervenciones de urgencia condicionadas por las necesidades de las obras que las motivaron. En segundo lugar, no contamos con suficiente información sobre el tipo de poblamiento rural existente en las áreas donde se localizan las necrópolis. Además, el hábitat campesino durante el período visigodo está definido por el empleo de materiales perecederos y por una cultura material más pobre y poco conocida, dificultando en gran medida su identificación. ${ }^{5}$ Así pues, no podemos definir en las cercanías de estas necrópolis la presencia de algunos de los asentamientos que parecen protagonizar los patrones de ocupación tardoantiguos en el territorio emeritense como vici o villulae (Cordero Ruiz 2011: 552-554).

En la península ibérica, algunas de las necrópolis mejor conocidas vinculadas con villae son las de Cabriana, La Olmeda, El Munts, El Ruedo y El Jardincillo (Chavarría Arnau 2007: 107-110). En estos yacimientos no se ha constatado la presencia de tumbas privilegiadas, lo cual puede relacionarse con la inhumación en otros lugares de los propietarios. Por

\footnotetext{
${ }^{4}$ Mencionadas en el Vitas Sanctorum Patrum Emeritensium (en lo sucesivo VPE) (VPE II, 21), todavía no han podido ser definida a nivel arqueológico. No obstante, parecen corresponderse con pequeñas explotaciones agrícolas - granjas - conformadas por almacenes y zonas de habitación (Isla Frez 2001: 15).

${ }^{5}$ Azkarate y Quirós Castillo (2001). De todas formas, se han hecho considerables avances en la detección y comprensión de los asentamientos rurales campesinos tardoantiguos y altomedievales, que han permitido afirmar la presencia de aldeas ya en periodos inmediatamente postromanos en otras zonas de la península ibérica, en consonancia con lo que se ha documentado en amplias regiones europeas. Tales datos y perspectivas aparecen recogidas en Quirós Castillo (2009).
} 


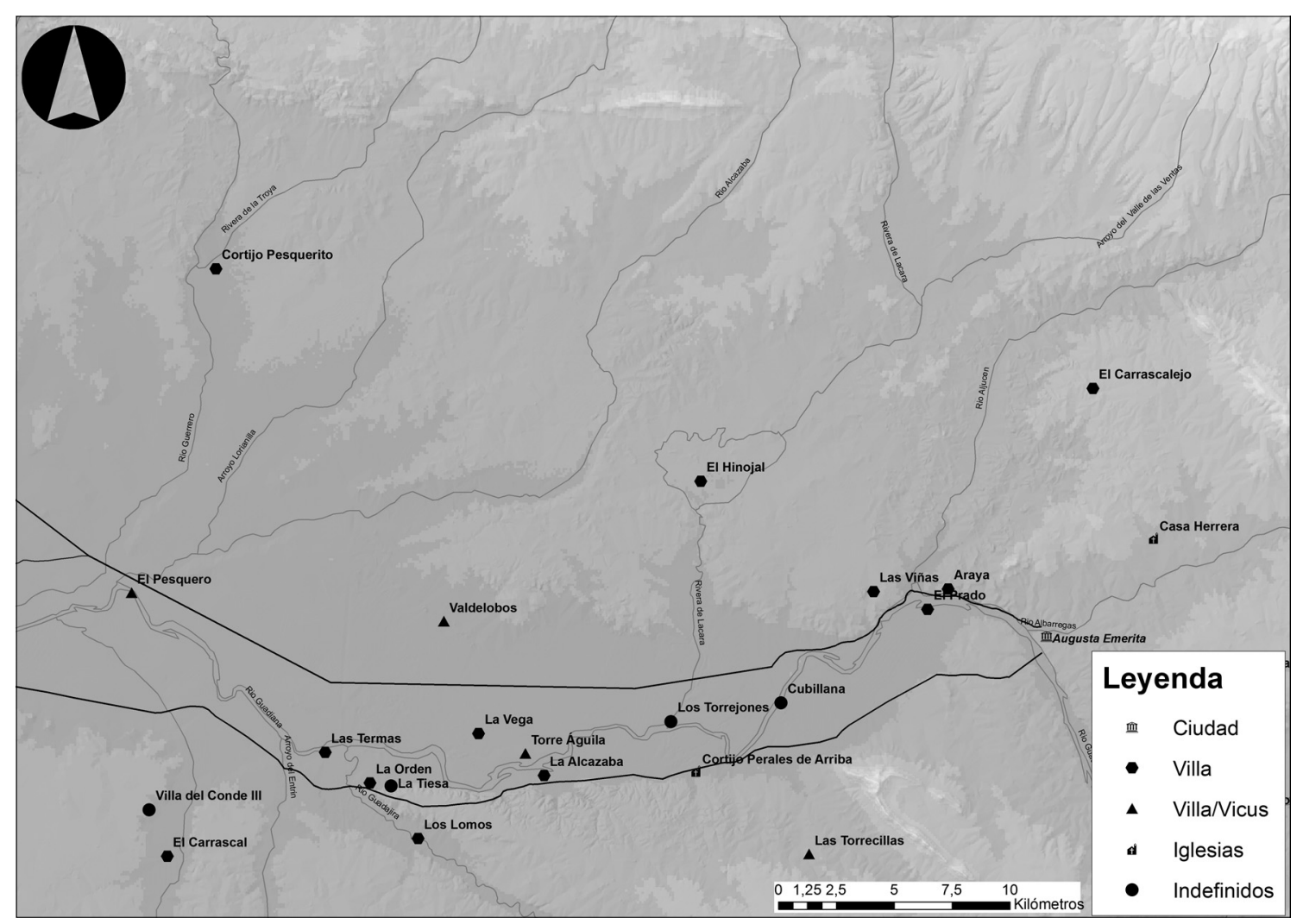

Figura 5. Principales yacimientos tardoantiguos conocidos en la cuenca media del Guadiana.

otro lado, su funcionamiento continuado durante la mayor parte de la Tardoantiguedad es relacionado con el mantenimiento de la propiedad a pesar del abandono de las villae como espacios de vivienda de sus propietarios. De esta manera, parece que los rustici y los domini vinculados a estos establecimientos siguen considerando estos cementerios como puntos de referencia, proponiéndose la relación entre esta población y los cambios funcionales de los sectores residenciales de estas villae a partir del siglo v (Chavarría Arnau 2007: 110). Además, no parece descabellado vincular la aparición de estos espacios funerarios con los procesos de concentración de la propiedad de la tierra y de la población rural iniciados a partir del siglo IV.

El registro material de Valdelobos se encuadra dentro de un territorio donde el final del sistema de villae a lo largo del siglo $\mathrm{v}$ dio paso a una realidad protagonizada por nuevos establecimientos rurales. Los patrones de ocupación todavía no están demasiados claros debido a la escasez de documentación. No obstante, parece que durante el período visigodo el poblamiento tendió a concentrarse en antiguas villae tardorromanas o en núcleos de nuevo cuño en las proximidades de antiguas calzadas ${ }^{6}$ y en un paisaje cristianizado (Fig. 5).

Por otro lado, si tomamos como referencia el registro material emeritense y lo comparamos con el documentado en otras zonas de la península (Chavarría Arnau 2007), puede plantearse que la edilicia de los asentamientos conocidos se caracterizaría, también, por el uso de de materiales reutilizados y perecederos. Sin embargo, queremos resaltar que no parece que esta evolución deba relacionarse con el cuadro de conflicto y decadencia defendido por la historiografía tradicional.

Las fuentes documentales señalan que, tras la conquista islámica, diferentes tribus bereberes se instalaron en el antiguo territorio emeritense durante el siglo vIII, aunque este proceso todavía no se ha constatado con seguridad (Franco Moreno 2011: 530-547). Los trabajos desarrollados en los yacimientos de Val-

${ }^{6}$ Un caso similar se ha podido detectar en la zona meridional de la actual Comunidad Autónoma de Madrid (Vigil-Escalera Guirado 2009). 
delobos y Cerro de las Baterías (La Albuera, Badajoz), parecen indicar que en la segunda mitad de esta centuria se está conformando un nuevo tipo de poblamiento rural que, por ahora, sólo alcanzamos a intuir (Cordero Ruiz y Franco Moreno 2012: 159-166). Por otro lado, conviene resaltar que no existe un registro material que induzca a pensar que la conquista islámica supuso un momento de ruptura en el territorio, sino el inicio de un nuevo modelo de asentamiento rural que paulatinamente sustituirá a los antiguos patrones de ocupación, bien ejemplificados en el yacimiento de Valdelobos.

\section{LA PIZARRA DE VALDELOBOS Y SU CONTENIDO}

La pizarra inscrita encontrada en el yacimiento de Valdelobos es un fragmento de $1535 \times 891 \times 120 \mathrm{~mm}$ con un color gris oscuro, casi negro (Fig. 6). Esta tipología y la ausencia de afloramientos pizarrosos en las proximidades del yacimiento nos lleva a proponer su procedencia en el área de los actuales términos municipales de Puebla de Obando (Badajoz) y Villar del Rey (Badajoz), núcleos localizados a unos 30 kilómetros al Norte. En esta zona abundan afloramientos pizarrosos de este tipo, caracterizados por la coloración ya referida y por presentar una superficie lisa y homogénea.

El fragmento conservado está escrito por una sola cara, aunque presenta dos cuentas diferentes, separadas por una línea vertical. Cada una de las inscripciones numéricas corresponde a una mano diferente (Fig. 7).

La situada a la izquierda $-y$ posiblemente la primera en ser realizada - tiene un ductus más grueso e inseguro, frente a una mayor seguridad y regularidad en el caso de la segunda. En el lado izquierdo se conservan 10 líneas, de las cuales aparecen completas 6 de ellas, las que corresponderían a las líneas $2,3,4,5$ y 6 y quizá la 8 . A continuación se transcriben dichas inscripciones numéricas:

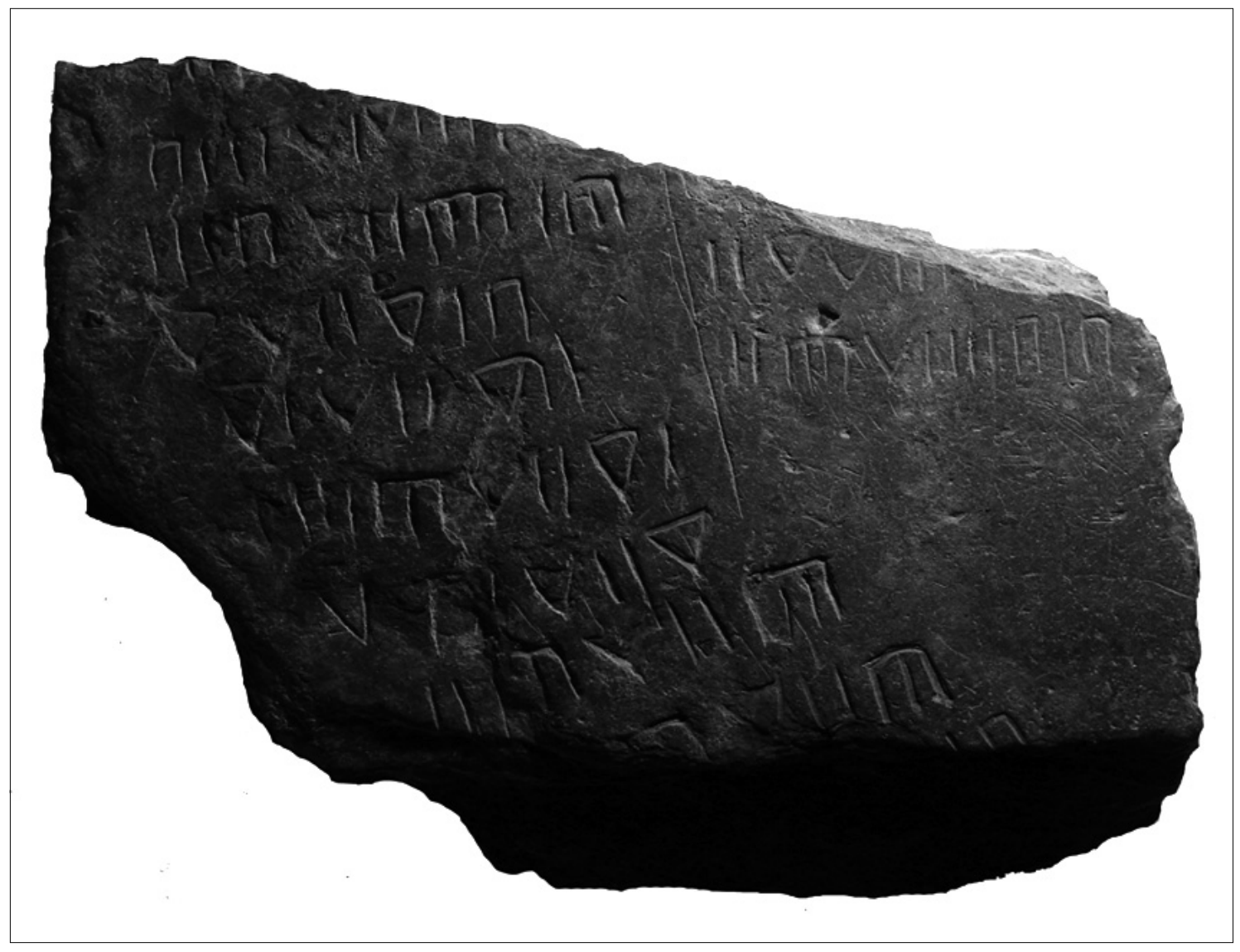

Figura 6. Pizarra de Valdelobos. 


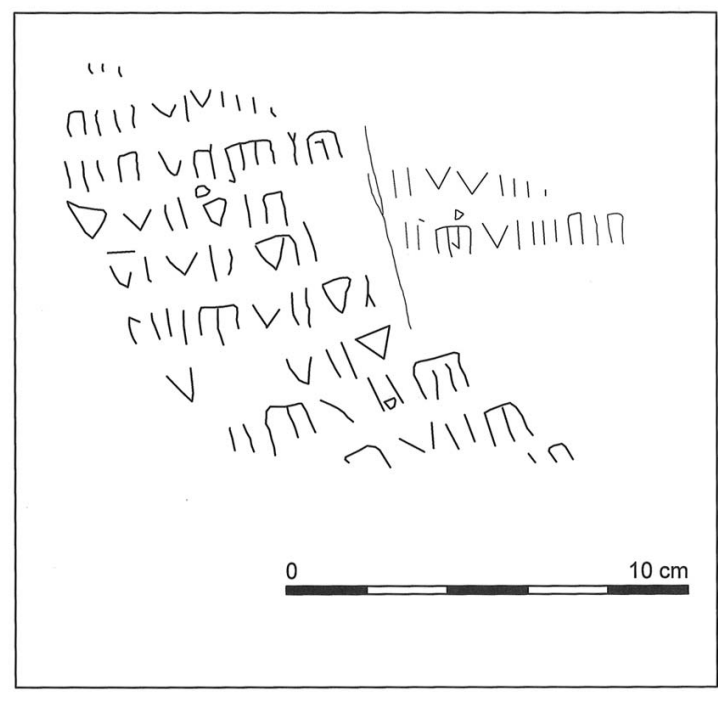

Figura 7. Transcripción propuesta de las cuentas identificadas en la pizarra.

1. I I I $(\ldots)^{7}$

2. II I II [V] I V I I I I

3. I I I II V II IIII I III

4. V V II V I II

5. V I V II VI I

6. I I I I III V II V I

7. $\mathrm{V}[\ldots] \mathrm{V}$ II $\mathrm{V}^{9}$

8. [...?] I I I II V I I IIII

9. [...] II V II III

10. $[\ldots]$ I II

Cada línea se compone de una serie de cantidades que se mueven entre 1 y 6 (línea 5), que se configuran en combinaciones variadas. Los sumandos que incorporan más de un signo (II, VI...) llevan en la parte superior una línea horizontal que junta dichos signos y que no ha podido representarse. Pero también algunos signos aislados con valor $\mathrm{V}$ disponen de esa línea superior (líneas 4, 5, 6, 7 y 8). Aunque resulta difícil la interpretación, debe plantearse que estemos ante una indicación por parte del escriba de que se trataba de una cantidad cerrada de 5 unidades, si bien se desconoce la causa de esa diferenciación con respecto a los signos $\mathrm{V}$ aislados que carecen de esa línea (líneas 2, 3, 4, 5, 6, 7 y 9).

\footnotetext{
${ }^{7}$ Línea muy mal conservada.

${ }^{8}$ El signo V intermedio está algo borroso. Parece completa, aunque los numerales finales solo se conservan fragmentariamente.

${ }^{9}$ Hay un raspado, que impide la lectura completa, aunque podría ser III.
}

La suma de las combinaciones de cada línea tiende a una cifra de 20 unidades. Así sucede con las líneas 2, 3, 4, 5 y 6, que se han conservado íntegramente. Es posible que la línea 7 también sumara esa cantidad, ya que el raspado ocupa un sitio que podría ser ocupado por un signo III. Esta circunstancia debe asociarse con el tipo de operación matemática que se recoge en estas piezas, compuesto por la suma de unidades desconocidas, a partir de líneas que dan como resultado un número constante, que podría ser luego multiplicado. Sin embargo, la línea 8 arroja un resultado diferente, pues únicamente se suman 16 unidades. Esta irregularidad podría ser debida a que falte la parte inicial de la línea, ya que la pizarra está rota en ese punto. Sin embargo, es una conjetura, ya que no hay signos de inscripciones numéricas siquiera fragmentarias ni parece existir espacio para un signo IIII.

Por otra parte, se conservan únicamente dos líneas en el lado derecho, que corresponden al final de una cuenta más extensa, cuya parte superior se ha perdido. La primera de ellas está incompleta, pero la segunda se ha conservado íntegramente. A continuación se ofrece la transcripción:

\section{II V V I I I [...] \\ 2. I I IIII V I I I I II I II}

Se aprecia de nuevo cómo las cantidades que configuran las líneas van desde I a V, es decir unidades no excesivamente grandes. Al igual que en el lado izquierdo, se observa el uso de un nexo entre signos mediante una línea horizontal superior que los une y parece, aunque no es seguro, que se utiliza también en un signo $\mathrm{V}$ aislado en la línea 1, concretamente el primero de ellos. Conviene resaltar que la cantidad sumada en la línea conservada íntegramente (20) es la misma que en la cuenta del lado izquierdo, lo que parece situarnos ante la continuidad de una operación matemática, pero en este caso realizada por otra persona.

Estas características se ajustan a lo que sabemos sobre el contenido de este tipo de inscripciones. ${ }^{10}$ En

\footnotetext{
${ }^{10}$ Descartamos, en cambio, la posibilidad de que se trate de un "ejercicio de escuela", como el que se ha documentado en Dehesa del Castillo (Diego Álvaro, Ávila) (Velázquez 2004: 296-299, $\mathrm{n}^{\circ}$ 58). Las diferencias son evidentes, ya que la pizarra encontrada en Valdelobos no posee texto y procede de un contexto vilicario o funerario; por el contrario, Dehesa del Castillo ha proporcionado un archivo que probablemente se relacione con un centro eclesiástico (Martín Viso 2006: 279), lo que explicaría mejor ese tipo de ejercicio, que figura junto a un salmo, es decir un texto religioso. En nuestra opinión, el uso de la pizarra como soporte escriturístico implica una pluralidad de funciones y de usos, que, en el caso de
} 
ellas se recoge un sistema matemático aparentemente sencillo, compuesto por líneas en las que aparecen combinaciones diferenciadas de cantidades que suman un número repetido línea tras línea, lo que favorecería la multiplicación. En el caso que nos ocupa, las cantidades tienden a sumar 20. Es una de las cifras que más se suele repetir, pues en el amplio corpus de El Cortinal de San Juan (Salvatierra de Tormes, Salamanca), con algo más de un centenar de piezas (Díaz y Martín Viso 2011), al menos ocho de ellas repiten esa cantidad en cada línea. De igual manera sucede en una pizarra numeral hallada en Quintanilla de Arriba (Valladolid) (Represa 1976). No obstante, hay otras muchas combinaciones que se han podido recoger que usan cifras diferentes. Las razones de la elección de una u otra cantidad son desconocidas, aunque puede conjeturarse que debían obedecer a la necesidad de contar en unidades superiores, subdivisibles en esos números. Sin embargo, ninguna de las medidas heredadas del mundo romano (modios, heminas, sextarios) se subdivide en 20 sino en 16 y 32. La existencia de una irregularidad en la línea 8 del lado izquierdo (que suma 16, lo que podría ser una cantidad en sextarios que llegarían a un modio) no es extraña, pues muchas de estas cuentas presentan estas disparidades puntuales. Podría tratarse de un error en el cálculo o quizá se trate de un ejercicio contable distinto.

La existencia de dos cuentas diferenciadas no es algo desconocido, aunque sí poco habitual, debido al estado muy fragmentado en el que se han conservado las piezas. Existen algunos ejemplos entre las pizarras procedentes de Salvatierra de Tormes (Salamanca) (Díaz y Martín Viso 2011). Sin embargo, en tales casos se aprecia la existencia de una diferencia clara en el contenido, ya que la segunda de las cuentas parece ser una sub-cuenta: la cuenta principal aparece cerrada y la cuenta secundaria es mucho más pequeña y está formada por líneas que suman cantidades inferiores. La particularidad de esta pizarra procede del hecho de que se trata de dos cuentas semejantes, incluso en el resultado de los sumatorios de cada línea, por lo que cabe sospechar que se usaron espacios vacíos en la cara de la pizarra para llevar a cabo un tipo de suma semejante. En tal sentido, estaríamos ante un indicio de la continuidad de la contabilidad que dio lugar a esta pieza, debido a que dos manos diferentes, en momentos distintos - aunque no necesariamente muy alejados en el tiem-

Valdelobos, se asemejan, como veremos, a lugares que proporcionan exclusivamente o de forma abrumadoramente predominante pizarras numerales y no de texto, tales como Lerilla, Salvatierra de Tormes o Cabeza de Navasangil. po- usaron el mismo sistema para contar algo que también debía ser idéntico.

Quedan pendientes algunas cuestiones sobre las que convendría profundizar o reflexionar. Desde luego, la principal es saber qué se contaba con este sistema. Esto resulta prácticamente imposible de saber en el caso de la pizarra procedente de Valdelobos. Solo puede certificarse que el sistema de cuenta exigía unos conocimientos mínimos de aritmética y de dominio de los objetos de escritura que ni estaban al alcance de todos ni eran necesarios para la mayoría.

\section{LOS HALLAZGOS DE PIZARRAS Y SU PROBLEMÁTICA}

Las inscripciones en pizarra han sido documentadas sobre todo en el sector sudoccidental de la cuenca del Duero (provincias de Ávila y Salamanca), así como en la vertiente meridional de las serranías del Sistema Central situadas en la actual provincia de Cáceres (Velázquez Soriano 2004: 40-43). En esta amplia región no solo se concentran abrumadoramente los hallazgos sino que, además, se documentan los principales yacimientos que han proporcionado el mayor número de piezas: Dehesa del Castillo (Diego Álvaro, Ávila), Cabeza de Navasangil (Solosancho, Ávila), Lerilla (Zamarra, Salamanca) y El Cortinal de San Juan (Salvatierra de Tormes, Salamanca) son los casos más significativos, aunque no los únicos (Díaz y Martín Viso 2011). Sin embargo, esta concentración no debe llevarnos a engaño, pues contamos ya con referencias al uso de este tipo de material en otros lugares. Los datos más actuales nos hablan de una dispersión de los hallazgos por amplias zonas de la cuenca del Duero (Represa 1976; Calleja Martínez 2001; Mañanes Pérez 2002; Regueras Grande y Martín Benito 1997), con especial relieve en el área segoviana, donde los centros de Virgen del Castillo (Bernardos) (Urbina Álvarez 2006; Gonzalo Gonzalo 2006) y sobre todo Cauca (Blanco García 2002; Pérez González y Reyes Hernández 2009b) son especialmente relevantes, dada su condición de puntos jerárquicos. Pero también al sur del Sistema Central, en la zona madrileña, se han efectuado hallazgos de algunas piezas en determinados asentamientos, tanto en las áreas serranas (Cancho del Confesionario, Manzanares el Real) (Caballero Zoreda y Megías Pérez 1977) como en los espacios sedimentarios (El Pelícano, Arroyomolinos) (Urbina Álvarez 2006). Los trabajos que se están llevando a cabo en Vega Baja, en Toledo, probablemente el lugar donde se encon- 
traba la sedes regia del regnum Visigothorum, también han documentado algún ejemplar. ${ }^{11}$ Incluso se conocen piezas que alcanzan localizaciones lejanas del foco principal, como sucede con el fragmento de una pizarra numeral encontrada en el sur de Portugal (Tente y Soares 2007) o la pizarra con dibujo procedente de Roc d'Enclar (Andorra) (Velázquez Soriano 1997).

La multiplicación de estos puntos nos habla de una tipología más extendida cuyo uso fue más amplio del que hasta ahora se había sospechado, por lo que la particularidad del área Salamanca-Ávila responde más a la intensidad de su utilización o a su mayor conservación, pero no a que su uso fuera una particularidad regional. Ahora bien, ninguna de las pizarras encontradas posee textos. ${ }^{12}$ Probablemente esta sea otra de las particularidades del foco central de hallazgos, aunque no debe olvidarse que la mayoría de las pizarras escritas, en torno a un 36\%, proceden del lugar de Dehesa del Castillo, en Diego Álvaro, que quizá fuera un centro eclesiástico cuyo archivo estaba escrito en pizarra. En cualquier caso, lo que parece seguro es que existía un uso escriturario en pizarra relativamente frecuente, cuya finalidad fundamental era contable, siguiendo un tipo de operación matemática que también se documenta en el Norte de África a finales del siglo $\mathrm{V}$, como se recoge en las denominadas tablillas Albertini (Courtais et alii 1952). Se trataría, como se comprueba en la pizarra de Valdelobos, de un sistema adaptado a una contabilidad constante de unidades desconocidas, de la que se podían fácilmente extraer cantidades mayores mediante la simple multiplicación de las líneas. A pesar de esa aparente sencillez, este sistema exigía una serie de conocimientos matemáticos que no estaban al alcance de cualquier persona durante este periodo y que sólo podían ser útiles para alguien que precisara de una contabilidad constante y que dispusiera de los medios para ello, lo que descarta una contabilidad de tipo campesino (Díaz y Martín Viso 2011).

Ahora bien, se plantea el problema de qué es exactamente lo que se cuenta y, derivado de él, quién está realizando estas cuentas. Teniendo en cuenta las apreciaciones anteriores, debe pensarse en dos posibilidades que no son excluyentes. Por un lado, que se tra-

${ }^{11}$ Noticia procedente www.europapress.es, con fecha de 20 de julio de 2009, consultada en www.arqueologiamedieval. com el 3 de agosto de 2009.

${ }^{12}$ Las pizarras con textos halladas en Carrio (Asturias), Huerta de los Frailes (León) y Fuente Encalada (Zamora) corresponden todas ellas a piezas de época posterior, datadas entre los siglos VIII y x (Diego Santos 1994: 27-29; Esparza y Martín Valls 1998). Agradecemos a Avelino Gutiérrez sus informaciones sobre la pizarra de Huerta de los Frailes. tara de una contabilidad de carácter dominical, en la que quedaran registradas cantidades relativas a la gestión de una gran propiedad, como podría ser el pago de censos por dependientes. Un uso de este tipo aparece atestiguado por las tablillas Albertini, que, al fin y al cabo, eran la contabilidad de un dominus. Pero cabe suponer que en otras ocasiones se estuviera ante una contabilidad de carácter fiscal, semejante a la que parece encubrirse en algunos documentos con textos, como los denominados por los editores vectigalia rerum rusticarum procedentes de Diego Álvaro, tal y como algunos estudios han puesto de manifiesto (Martín Viso 2006). Esta explicación se ajustaría mejor a aquellos casos en los que las pizarras han aparecido en contextos donde no aparece claramente una presencia dominical, como sucede con determinados centros rurales que emergen a partir del siglo V como auténticos ejes del paisaje rural en el suroeste de la meseta del Duero (Martín Viso 2008b; Díaz y Martín Viso 2011). Ambas interpretaciones son factibles y no se excluyen entre sí, dado que el sistema contable pudo utilizarse en situaciones diversas, pero también porque los claros límites que diferenciaban el impuesto de la renta dominical se van desvaneciendo tras el final de la estructura imperial romana en la pars Occidentalis (Wickham 2005), por lo que el dominus podría ser también recaudador de tributos o atribuirse esa función y convertirlos en parte del censo campesino.

Un conocimiento más ajustado de los usos de esta contabilidad solo puede realizarse a través de la existencia de contextos arqueológicos más o menos bien definidos que nos permitan integrar las pizarras en cada yacimiento. Por desgracia, la mayoría de los hallazgos carecen de ese contexto, bien porque se produjeron en unas circunstancias en las que únicamente interesaba el descubrimiento del objeto en sí, como sucede en muchas intervenciones hasta mediados del siglo $\mathrm{xx}$, bien porque han aparecido realmente fuera de contexto o en una posición secundaria, como sucede en Virgen del Castillo (Urbina Álvarez 2002). Únicamente algunos casos muy específicos parecen ofrecernos una información más clara. Así, en Ávila, en el solar del Episcopio, las pizarras aparecen en un contexto en el que también se documentan cerámicas a torno estampilladas y un tremis de oro de Chindasvinto (Díaz de la Torre 2003). Todo apunta a un lugar con un cierto nivel social y económico que quizá se asocie a la captura fiscal, por la presencia de la moneda (Martín Viso 2008a). Por otro lado, en Monte el Alcaide (Monleón, Salamanca), las pizarras se encontraron asociadas a una pileta de unos 28 litros, situadas en una posición que hace pensar que 
fueron depositadas conscientemente de esa forma (Paricio Alonso y Vinuesa Chao 2009). La existencia únicamente de cerámicas de almacenamiento hace pensar que este lugar tenía una funcionalidad como punto de captación de un producto, probablemente vino, que se elaboraba en las inmediaciones. Esta circunstancia recuerda, si bien en una escala menor, a lo que se ha documentado en la región actualmente tunecina de Kasserine durante el periodo tardoantiguo, donde existirían grandes depósitos destinados a concentrar la producción de aceite de los alrededores (Hitchner 1995). Por último, en Cabeza de Navasangil (Solosancho, Ávila), las pizarras encontradas no lo fueron durante las campañas de excavación de los años setenta y noventa, pero se vinculan al arrasamiento por fuego del lugar, una circunstancia datada entre los siglos VI y VII (Larrén 1989; Larrén et alii. 2003). Interesa destacar como nos encontramos con un pequeño punto fortificado emplazado a media ladera de la sierra de la Paramera, en una posición dominante sobre el valle Amblés, lo que hace pensar en un castellum en manos de elites locales, ante la ausencia de evidencias sobre la presencia efectiva de un poder central (Martín Viso 2008b: 235236). Estos tres casos ejemplifican la diversidad de posibles contextos en los que se han hallado pizarras, y que pueden entenderse respectivamente como la consecuencia de usos fiscales, dominicales o asociados al control de un paso ganadero. No obstante, todos ellos tienen un denominador común: se trata de espacios de poder político, económico y social, alejados de contextos campesinos o artesanos.

Por otra parte, se acepta comúnmente que estas pizarras pertenecen a la época visigoda. El hecho de que hayan aparecido algunas piezas en las que se han conservado textos escritos y el sistema contable ya descrito, ha permitido colegir una unidad temporal de ambas tipologías, atendiendo al estudio paleográfico de las pizarras escritas, algunas de las cuales tienen indicaciones directas de su datación en los siglos VI y VII. De esta forma, las pizarras se han convertido en un 'fósil director' que fecharía un contexto estratigráfico e incluso un yacimiento, ante la ausencia de otros parámetros más inequívocos.

Sin embargo, casi todas las pizarras numerales encontradas se han recogido fuera de posición estratigráfica, lo que supone una dificultad a la que se une la constatación del uso de la pizarra como soporte de textos privados en la época del Principado (Gimeno Pascual y Stylow 2007) y la posibilidad de que algunas de las pizarras numerales sean de época tardorromana. El caso más elocuente es la pizarra encontrada entre el material de un basurero en el ya- cimiento de San Pelayo (Aldealengua, Salamanca) datado entre finales del siglo IV y comienzos del v. Se da la circunstancia de que dicho lugar ha sido identificado con una villa periurbana de la civitas de Salmantica (Dahí Elena 2007), por lo que no resulta arriesgado pensar en una contabilidad asociada a las necesidades del dominus, bien referida a pagos de censos o a algún tipo de actividad doméstica. Algo semejante sucede en Cauca, donde se conocen al menos dos focos de hallazgos de pizarras. Uno se encuentra en la zona conocida como Los Azafranales, junto al casco urbano tardorromano, un lugar utilizado en ese periodo para verter basuras domésticas. Aquí se ha encontrado un conjunto de pizarras numerales, diez de las cuales se consignaron en un artículo con una localización errónea (Sáez 1985), a las que se deben sumar cerca de medio centenar en manos de particulares (Blanco García 1998 y 2002: 169). Algunas de ellas han sido recientemente publicadas, una de las cuales se halló en la colmatación de una fosa-basurero, mientras otras dos fueron encontradas en el nivel de arada y en el interior de de la colmatación de una gran zanja que sirvió para extraer elementos de un muro romano con una cronología muy amplia, pues las cerámicas encontradas llegan al periodo bajomedieval (Pérez González y Reyes Hernando 2009b: 50-54). Aunque los datos son inseguros, parece razonable pensar que estamos ante los restos de una contabilidad efectuada en época tardorromana. El otro foco se sitúa en el pago denominado Tierra de las Pizarras o Las Pizarras, un área suburbana donde existió una residencia lujosa, que puede calificarse como una villa. Este lugar parece haber sido abandonado a partir del siglo v d.C., cuando buena parte de los materiales fueron reaprovechados, al mismo tiempo que se diseñó un espacio de enterramientos, subdivido en dos áreas (Pérez González y Reyes Hernández 2008 y 2009a). Es en este punto donde apareció una pizarra, fragmentada en 6 trozos, como consecuencia de una actividad de unos furtivos, por lo que carecemos de una imagen clara de su contexto específico (Pérez González y Reyes Hernández 2009b: 54-56). Sin embargo, el hecho de que este espacio residencial no haya perdurado más allá del siglo v con esa función permite afirmar que estaríamos ante una pizarra de época tardorromana, asociada posiblemente a una contabilidad dominical.

Estos datos desvelan la posible existencia de pizarras con dataciones tardorromanas, si bien el hecho de haberse encontrado en contextos secundarios, incluyendo basureros, representa una dificultad para ir más allá del terreno de la hipótesis. En cambio, las 
excavaciones efectuadas en el edificio del Episcopio en Ávila han detectado la presencia de fragmentos de pizarras numéricas en estratos de época post-romana, lo que certifica que tales piezas se usaron en esos momentos (Díaz de la Torre 2003). Todo ello parece encaminarse hacia la idea de un periodo de uso de estas pizarras numerales más dilatado de lo que se ha propuesto tradicionalmente y que se iniciaría al menos ya en el siglo IV. Por consiguiente, las pizarras no pueden datar sin más una fase en una secuencia estratigráfica, sino que solo son comprensibles en su contexto, que nos ofrecerá las claves para dar una cronología. En tal sentido, la comprensión de los contextos específicos de hallazgos es indispensable, tanto para obtener cronologías fiables como para entender los posibles usos.

¿Qué puede aportarnos en tal sentido la pizarra de Valdelobos? Por un lado, su conocimiento nos pone nuevamente de relieve la amplitud del uso del sistema contable ya descrito, que debía extenderse por toda Hispania y por buena parte del Mediterráneo occidental, superando el marco regional del suroeste de la meseta del Duero. Por tanto, el uso de la pizarra como material escriturístico no es una particularidad regional, aunque sí lo es su extraordinaria intensidad y su aplicación al registro de documentación privada escrita. Por otro lado, cabe destacar que se trata de la primera pizarra numeral hallada en el ager Emeritensis. Hasta ahora, solo conocíamos un único ejemplar localizado en la ciudad, procedente de las cercanías de la necrópolis periurbana de Los Columbarios. En esta pizarra se han identificado dos dibujos: la planta repetida de un edificio de planta basilical con una cabecera con dos ábsides y una crátera de pie triangular de doble asa. Esta última presenta evidentes concomitancias con representaciones decorativas halladas en las Hayes 76 tipo E, datadas entre los siglos v y vi (Mateos Cruz 1997: 59). Los dos motivos representados llevaron a Mateos Cruz a relacionar esta pizarra con una tabla de instrucción similar a las documentadas por Velázquez Soriano (2005b: 118-123), aunque, también, cabe la posibilidad de incluirla dentro del grupo con diseños de labores agrícolas y edificaciones religiosas (Santonja y Moreno 1991-1992).

El uso de la pizarra como soporte escriturístico, aunque no propiamente local —los posibles lugares de extracción de pizarra de Valdelobos se encuentran a unos 30 kilómetros al norte_-, quizá se explique por una oferta insuficiente de papiro o por un menor coste en el caso de la pizarra. Sea como fuere parece que había una tradición de utilización de la piza- rra como soporte para la escritura que estaría extendida por amplios sectores de la península ibérica, aunque es probable que estuviera asociada a su uso para una documentación que no estaba destinada a su perduración, con la notable excepción del área principal de los hallazgos. Por otro lado, la pizarra sería un indicio de la existencia de lazos comerciales en el seno de ager Emeritensis, que posibilitan la obtención del material de escritura.

Por lo que se refiere a la cronología de la pizarra numeral de Valdelobos nos parece plausible, a pesar de que los datos no son demasiados precisos, presentar como hipótesis de trabajo una datación encuadrada entre los siglos IV y v, vinculando su función contable con alguna de las dos últimas fases de uso del almacén descrito anteriormente. La más que probable presencia de la pars urbana de una villa al norte de esta, seguramente el núcleo del fundus en el que se insertaba el dicho almacén, parece indicarnos que la contabilidad escrita en la pizarra es de carácter dominical, relacionándose las cantidades reseñadas con la gestión de esta propiedad. Esta idea también se fundamenta en la total ausencia de indicios - políticos, económicos o sociales- datados entre los siglos VI y VII que nos permitan suponer la realización de actividades necesitadas de una con-tabilidad. Por otro lado, cabe recordar que, al igual que en el caso de otras necrópolis tardoantiguas localizadas en las cercanías de Valdelobos anteriormente mencionadas, no disponemos de datos que nos permitan asegurar un poblamiento de esta cronología, ya sea concentrado o disperso, en su entorno inmediato.

El principal problema de esta hipótesis de trabajo es que la pizarra se halló en un contexto secundario, lo cual nos impide corroborarla de manera inmediata. No obstante, los datos desprendidos del contexto general del yacimiento y del territorio donde se inserta la pieza estudiada parecen avalar nuestra teoría, más si tenemos en cuenta que los casos de San Pelayo y de Cauca presentan características semejantes a las que aquí describimos. Sin embargo, no podemos descartar definitivamente que la pizarra numeral de Valdelobos se date entre los siglos vi y vII, aunque esta hipótesis no nos parezca la más adecuada debido a la falta de datos concretos que la avalen. Además, como ya hemos mencionado, no podemos usar sin más las pizarras como fósil director o como prueba incuestionable para encuadrar una secuencia ocupacional determinada o, incluso, un yacimiento en época visigoda ante la ausencia de otras pruebas arqueológicas. 


\section{BIBLIOGRAFÍA}

Azkarate Garai-Olaun, A. y Quirós Castillo, J. A. 2001: "Arquitectura doméstica altomedieval en la península ibérica. Reflexiones a partir de las excavaciones arqueológicas de la catedral de Santa María de Vitoria-Gasteiz, País Vasco", Archeologia Medievale 28, 25- 60.

Blanco García, J. F. 1998: "Aproximación a la Cauca del bajo imperio”, R. Teja y C. Pérez González (eds.), Congreso Internacional La Hispania de Teodosio 2, Valladolid-Segovia, 377-393.

Blanco García, J. F. 2002: "Coca, Cauca”, T. Mañanes (dir.), Arqueología del área central de la cuenca del río Duero: de Simancas a Coca, Valladolid, 127-173.

Caballero Zoreda L. y Megías Pérez, G. 1977: “Informe de las excavaciones del poblado medieval del Cancho del Confesionario, Manzanares el Real (Madrid), Noticiario Arqueológico Hispánico 5, 325-331.

Calleja Martínez, M. ${ }^{a}$ V. 2001: "El poblamiento de época visigoda en el sureste de la provincia de Valladolid" , V Congreso de Arqueología Medieval Española 1, Valladolid, 125-129.

Chavarría Arnau, A. 2007: El final de las villae en Hispania (siglos IV-VIII), Turnhout.

Cordero Ruiz, T. 2010: "Una nueva propuesta sobre los límites del ager Emeritensis durante el Imperio Romano y la Antigüedad Tardía", Zephyrus 65, 149-165.

Cordero Ruiz, T. 2011: "El territorio emeritense durante la Antigüedad Tardía”, J. M. Álvarez Martínez y P. Mateos Cruz (eds.), 1910-2010. El Yacimiento Emeritense, Mérida, 547-562.

Cordero Ruiz, T. y Franco Moreno, B. 2012: "El territorio emeritense durante la Antigüedad Tardía y la Alta Edad Media”, L. Caballero Zoreda, P. Mateos Cruz y T. Cordero Ruiz (eds.), Visigodos y Omeyas V: El territorio, Mérida, 147-170.

Courtois, Ch. et alii. 1952: Tablettes Albertini. Actes privés de l'époque vandale (fin du v siècle), Paris.

Dahí Elena, S. 2007: "Un contexto cerámico de la Antigüedad tardía: el yacimiento de San Pelayo (Aldealengua, Salamanca). Nuevos datos sobre la cronología de las pizarras visigodas", Pyrenae 381, 79-104.

Díaz, P. C. y Martín Viso, I. 2011: "Una contabilidad esquiva: las pizarras numerales visigodas y el caso de El Cortinal de San Juan (Salvatierra de Tormes, Salamanca)", P. C. Díaz y I. Martín Viso (eds.), Between tax and rent. Fiscal problems from Late Antiquity to Early Middle Ages / Entre el im- puesto y la renta. Problemas de la fiscalidad tardoantigua y altomedieval, Bari, 221-250.

Díaz de la Torre, J. 2003: Informe final ( $1^{a}$ y $2^{a}$ fase) de los trabajos arqueológicos efectuados en el entorno del episcopio (Ávila de los Caballeros). Memoria inédita depositada en el servicio de patrimonio provincial, Ávila.

Diego Santos, F. 1994: Inscripciones medievales de Asturias, Oviedo.

Esparza Arroyo, Á. y Martín Valls, R. 1998: "La pizarra altomedieval de Fuente Encalada (Zamora): contribución al estudio de las inscripciones profilácticas", Zephyrus 51, 237- 262.

Franco Moreno, B. 2011: De Emerita a Mārida. El territorio de Mérida en el tránsito de la Antigüedad Tardía a la Edad Media, Saarbücken.

García y Bellido, A. 1965: "La villa romana del El Carrascal, Talavera la Real", Archivo Español de Arqueología 38, 83-96.

Gimeno Pascual, H. y Stylow, A. U. 2007: "Pizarras inscritas del Cerro del Moro y de la Marismilla", J. A. Pérez Macías y A. Delgado Domínguez (eds.), Las minas de Riotinto en época Julio Claudia, Huelva, 185-191.

Gonzalo Gonzalo, J. M. ${ }^{\text {a }}$ 2006: El Cerro del Castillo, Bernardos (Segovia). Un yacimiento singular en la provincia de Segovia durante la Antigüedad Tardía, Segovia.

Gorges, J. G. y Rodríguez Martín, F. G. 2000: "Voies romaines, propriétés et propiétaires à l'ouest de Mérida; problèmes d'occupation du sol en moyenne vallée du Guadiana sous le Haut-Empire", J. G. Gorges y T. Nogales Basarrate (eds.), Sociedad y cultura en la Lusitania romana, Badajoz, 101-155.

Hitchner, R. B. 1995: "Historical text and archaeological context in Roman North Africa: the Albertini Tablets and the Kasserine survey", D. B. Small (ed.), Methods in the Mediterranean: historical and archaeological views on texts and archaeology, Leiden, 124-142.

Larrén Izquierdo, H. 1989: "Materiales cerámicos de La Cabeza: Navasangil (Ávila)", Boletín de Arqueología Medieval 3, 53-74.

Larrén Izquierdo, H. et alii. 2003: "Ensayo de sistematización de la cerámica tardoantigua en la cuenca del Duero", L. Caballero, P. Mateos y M. Retuerce (eds.), Cerámicas tardorromanas y altomedievales en la Península Ibérica: ruptura y continuidad, Madrid, 273-306.

Mañanes Pérez, T. 2002: Arqueología del área central de la cuenca del río Duero: de Simancas a Coca, Valladolid. 
Márquez Pérez, J. 2007: Los Columbarios: arquitectura y paisaje funerario en Augusta Emerita, Ataecina 2, Mérida.

Martín Viso, I. 2006: “Tributación y escenarios locales en el centro de la Península Ibérica: algunas hipótesis a partir del análisis de las pizarras visigodas", Antiquité Tardive 14, 263-290.

Martín Viso, I. 2008: "Tremisses y potentes en el nordeste de Lusitania (siglos VI-VII)", Mélanges de la Casa de Velázquez 38-1, 175-200.

Martín Viso, I. 2008b: "La ordenación del territorio rural y la tributación en el suroeste de la meseta del Duero (siglos VI-VII)", S. Castellanos y I. Martín Viso (eds.), De Roma a los bárbaros. Poder central y horizontes locales en la cuenca del Duero, León, 227-261.

Mateos Cruz, P. 1997: "Representación basilical en una pizarra emeritense de época visigoda", Mérida: ciudad y patrimonio 1, 55-64.

Mateos Cruz, P. 2003: "Villa de Torre Águila. Barbaño", P. Mateos y L. Caballero (eds.), Repertorio de Arquitectura Cristiana en Extremadura: Época tardoantigua y altomedieval, Mérida, 115-123.

Matesanz Vera, P. y Sánchez Fernández, C. 2007: "Intervención arqueológica en la Finca Céspedes (Ferial de Badajoz, Lusiberia)", Jornadas sobre Arqueología de la ciudad de Badajoz, Badajoz, 125-167.

Paricio Alonso, F. y Vinuesa Chao, M. 2009: "Monte el Alcalde: un yacimiento altomedieval en el alto valle del Alagón (Monleón, Salamanca)", I. Martín Viso (ed.), ¿Tiempos oscuros? Territorio y sociedad en el centro de la Península Ibérica (siglos VII- X), Madrid, 45-67.

Pérez González, C. y Reyes Hernández, O. 2008: "Proyecto de investigación Las Pizarras (Coca, Segovia): campaña de investigación arqueológica del año 2007", Oppidum. Cuadernos de Investigación 4, 133-172.

Pérez González, C. y Reyes Hernández, O. 2009a: "Proyecto de investigación Las Pizarras (Coca, Segovia): campaña de investigación arqueológica del año 2008", Oppidum Cuadernos de Investigación 5, 7-38.

Pérez González, C. y Reyes Hernández, O. 2009b: "Epigrafía caucense (II)", Oppidum. Cuadernos de Investigación 5, 39-64.

Quirós Castillo, J. A. 2009: The archaeology of early medieval villages in Europe, Bilbao.

Ramírez Sádaba, J. L. 1991: "La inscripción de Torrebaja, (Pueblonuevo del Guadiana, Badajoz). Original modelo de la epigrafía cristiana", Antigüedad y Cristianismo 8, 89-98.
Regueras Grande F. y Martín Benito, J. I. 1997: "Hallazgos arqueológicos en el área de Benavente", Brigecio 7, 310-311.

Represa, M. ${ }^{a}$ F. 1976: "Una pizarra visigótica del tipo Lerilla”, Boletín del Seminario de Estudios de Arte y Arqueología 42, 452-457.

Rodríguez Martín, F. G. 1996: "La cerámica de paredes finas en los talleres emeritenses", Mélanges de la Casa de Velázquez 32, 139-179.

Rodríguez Martín, G. (1997): "La villa romana de Torre Águila (Barbaño, Badajoz) a partir del siglo IV d. C. Consideraciones generales”, R. Teja y C. Pérez González (eds.), Congreso Internacional la Hispania de Teodosio 2, Valladolid-Segovia, 697-712.

Rubio Muñoz, A. 1982: "La necrópolis hispano-visigoda de la Picuriña”, VII Congreso de Estudios Extremeños, Badajoz, 110-115.

Sáez, C. 1985: "Hallazgo de pizarras visigodas cifradas en Coca de la Vega", Anuario de Estudios Medievales 15, 35-37.

Santonja, M. y Moreno, M. 1991-1992: “Tres pizarras con dibujos de época visigoda en la provincia de Salamanca", Zephyrus 44-45, 471496.

Tente, C. y Soares, A. M. 2007: "Uma pizarra visigoda com inscrição numérica em Santa Margarida (Serpa)", Arqueologia Medieval 10, 13-19.

Urbina Álvarez, A. 2002: "Hallazgo de dos pizarras con inscripción en el hábitat Tardoantiguo del Cerro de la Virgen del Castillo (Bernardos, Segovia)", Faventia 24-1, 135-144.

Urbina Álvarez, A. 2006: "Pizarras con inscripción de época hispanovisigoda en la Provincia de Madrid", J. Morín de Pablos (ed.), La investigación arqueológica de la época visigoda en la comunidad de Madrid 3, Alcalá de Henares, 781786.

Velázquez Soriano, I. 1997: "Inscripció sobre pissarra”, VV.AA. (eds.), Roc d'Enclar. Transformacions d'un espai dominant, segles IV-XIX, Andorra, 418-422.

Velázquez Soriano, I. 2004: Las pizarras visigodas (Entre el latín y su disgregación. La lengua hablada en Hispania, siglos VI-VIII), Burgos.

Velázquez Soriano, I. 2005a: "La geografía de las pizarras: problemas planteados", I. Velázquez y M. Santonja (eds.), En la Pizarra. Los últimos hispanorromanos de la Meseta, Burgos, 93109.

Velázquez Soriano, I. 2005b: “Textos escritos en pizarra: la otra manifestación de la cultura escrita en la Hispania visigoda”, I. Velázquez y M. San- 
tonja (eds.), En la Pizarra. Los últimos hispanorromanos de la Meseta. Burgos, 111-126.

Vigil-Escalera Guirado, A. 2009: "Las aldeas altomedievales madrileñas y su proceso formativo", Qui- rós Castillo, J.A. (ed.), The archaeology of early medieval villages in Europe, Bilbao, 315-339.

Wickham, C. 2005: Framing the early Middle Ages. Europe and the Mediterranean, 400-800, Oxford.

Recibido: 11-05-2011

Aceptado: 15-09-2011 